\title{
LASER-MICROMACHINED DEFECT ARRAYS FOR DC POTENTIAL DROP FATIGUE STUDIES
}

\author{
C. B. Arnold ${ }^{* 1}$, B. Pratap ${ }^{2}$, A. Piqué ${ }^{2}$, A. B. Geltmacher ${ }^{3}$, and J. P. Thomas ${ }^{3}$ \\ ${ }^{1}$ Department of Mechanical and Aerospace Engineering, Princeton University, Princeton, NJ, USA \\ ${ }^{2}$ Materials and Sensors Branch, Naval Research Laboratory, Washington, DC, USA \\ ${ }^{3}$ Multifunctional Materials Branch, Naval Research Laboratory, Washington, DC, USA
}

\begin{abstract}
The experimental characterization of fatigue crack initiation and growth of structural materials can be very expensive and time consuming. Fatigue specimens are typically controlled by a single dominant defect and several specimens are needed to examine the fatigue response for each loading condition of interest. Time and expense add up as millions of load cycles are sometimes required to initiate a crack, and replicate tests are necessary to characterize the inherent statistical nature of fatigue. In order to improve the efficiency of experimentation, we are developing laser-based techniques to produce fatigue test samples with arrays of defects. Controlled arrays of oval shaped micro-defects are laser-micromachined in titanium alloy (Ti-6Al-4V). Crack initiation from the individual defects in the arrays is monitored using a DC potential drop technique. Results indicate the utility of this approach in multiplying the amount of fatigue data generated per specimen-test. The new fatigue test approach is applicable to a wide range of material systems and initial defect structures.
\end{abstract}

\section{INTRODUCTION}

Failure of a material via fatigue is an important consideration in structural design determining its applicability to a particular structural application [1,2]. Fatigue crack initiation under cyclic loading conditions is probabilistic in nature and requires multiple tests in order to develop a statistical sampling for a given material [3]. These tests can be quite time consuming and expensive, since a large number of load cycles may be required to initiate a crack from a single defect in each specimen. The acquisition of a single data point from each experimental test, which can take several days to perform, also increases the time needed for the required tests. In order to improve the efficiency of laboratory fatigue testing, a method that can provide multiple fatigue crack initiation defects on a single test specimen is necessary.

There are a number of experimental techniques used to monitor the fatigue "damage" present in a test sample. Potential drop (PD) methods (both DC and $\mathrm{AC}$ ) can provide an indirect measurement of crack initiation and growth from a geometric defect introduced in the specimen under fatigue loading $[2,4]$. In these methods, electrical current is passed through the specimen, and the change in electrical potential across the defect (typically a hole or notch in the specimen) is measured and correlated with the crack length [4]. As a crack initiates and grows, the electrical resistance between the potential drop leads increases leading to an increase in measured potential under constant current conditions. Obviously, one must know where the fatigue crack will initiate and grow in the specimen in order to use these methods. Typically, geometric defects such as holes and notches are used to produce a concentration of stress at their edges that provides a driving force for crack initiation. A statistical sampling of fatigue behavior can thus be accomplished by producing an array of defects that are individually monitored by potential drop probes at each defect. Arrays of defects can be generated relative to specific material microstructural features to combinatorially generate fatigue data relevant to micromechanical modeling and analysis.

In this paper, laser-based techniques for producing fatigue test samples with an array of elliptical holes is examined. Laser micromachining is used to create the array of small elliptical holes in a titanium alloy specimen while laser direct-

\footnotetext{
${ }^{*}$ Corresponding Author: cbarnold@princeton.edu; phone 609-258-0250; fax: 609-258-5877
} 
write is used to deposit precisely located potential drop lead attachments on opposite edges of the holes [5]. Fatigue testing of the test sample indicates that these defect arrays are capable of providing replicate fatigue data in a single fatigue specimen. These techniques form the basis of a combinatorial approach to fatigue testing.

\section{EXPERIMENT}

Laser-based techniques are used to produce defect arrays and create ohmic contacts on the fatigue sample. The main sample is a titanium (Ti-6 Al-4 V) "dog-bone" $200 \mathrm{~mm}$ long x $0.8 \mathrm{~mm}$ thick with a reduced gauge region of $76 \mathrm{~mm}$ long $\mathrm{x} 13 \mathrm{~mm}$ wide. The radius of curvature is $13 \mathrm{~mm}$. A pulsed UV laser with wavelength of $355 \mathrm{~nm}$ and duration of $30 \mathrm{~ns}$ (Nd: $\mathrm{YVO}_{4}$, Spectra Physics) is used as a source for these experiments. The laser pulse passes through a series of focusing optics and a UV microscope objective (typical spot size of $25 \mu \mathrm{m}$ ) before reaching the sample that is mounted on a vacuum chuck. Computer control of the sample position is accomplished using an $\mathrm{x}-\mathrm{y}$ motion control system (Aerotech D500) with inline video imagery to enable sample alignment and real time monitoring of the micromachining and material deposition process. Accurate control of the micromachined structures is obtained by varying the distance between subsequent laser pulses impinging on the sample.

For the potential drop technique to work, the metal sample must be electrically isolated from the electrical leads except in the region of the crack. Figure 1 shows a cartoon of the sample structures used to accomplish this. A composite of 50 $\mu \mathrm{m}$ acrylic based adhesive and $50 \mu \mathrm{m}$ thick liquid crystalline polymer (LCP) with $18 \mu \mathrm{m}$ thick electrodeposited copper is used to provide electrical conductivity to different regions on the sample and electrically isolate the sample from these conductors. The copper is etched using standard photolithographic techniques (Shipley S-1818 positive photoresist) and ferric chloride etchant to form parallel lines $1 \mathrm{~mm}$ wide spaced by $3 \mathrm{~mm}$. Along these lines, $2 \mathrm{~mm} \times 2 \mathrm{~mm}$ solder pads are patterned to provide means of electrical contact off the sample.
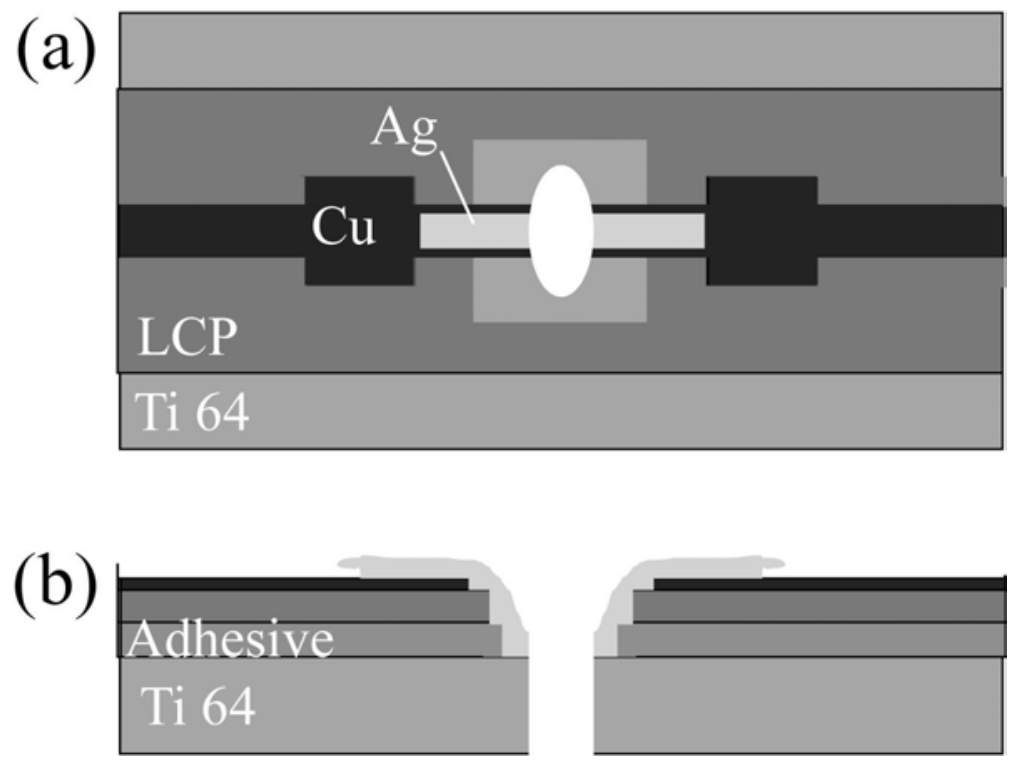

Figure 1: (a) Top-view of sample structure, (b) cross section view of sample structure.

This pattern is then coated with a surface protective coating (SPC) solution (Shipley FSC-L). The coating reduces debris adhesion to LCP and copper surfaces during subsequent laser processing. The SPC solution is spin coated at 3000RPM for 30 seconds on the patterned LCP. Upon baking for 30 minutes at $100^{\circ} \mathrm{C}$, an even layer of SPC covers the LCP-copper composite. A $50 \mu \mathrm{m}$ thick double-sided acrylic based adhesive (3M F9460PC) with polycoated kraft lining is then adhered to the back of the LCP composite. 
Rectangular shaped windows $4 \mathrm{~mm} \times 2 \mathrm{~mm}$ are laser micromachined in this LCP-acrylic based adhesive composite adjacent to the patterned copper lines to allow visual inspection of the Ti substrate during testing. These windows are machined at $45 \mathrm{~J} / \mathrm{cm}^{2}$. The paper backing is then removed and the LCP composite is aligned and placed on the Ti sample. The long edge of the window is aligned parallel with the loading axis of the sample. The acrylic based adhesive is cured in a furnace at $100^{\circ} \mathrm{C}$ for 1 hour under $0.8 \mathrm{kPa}$ mechanical pressure.
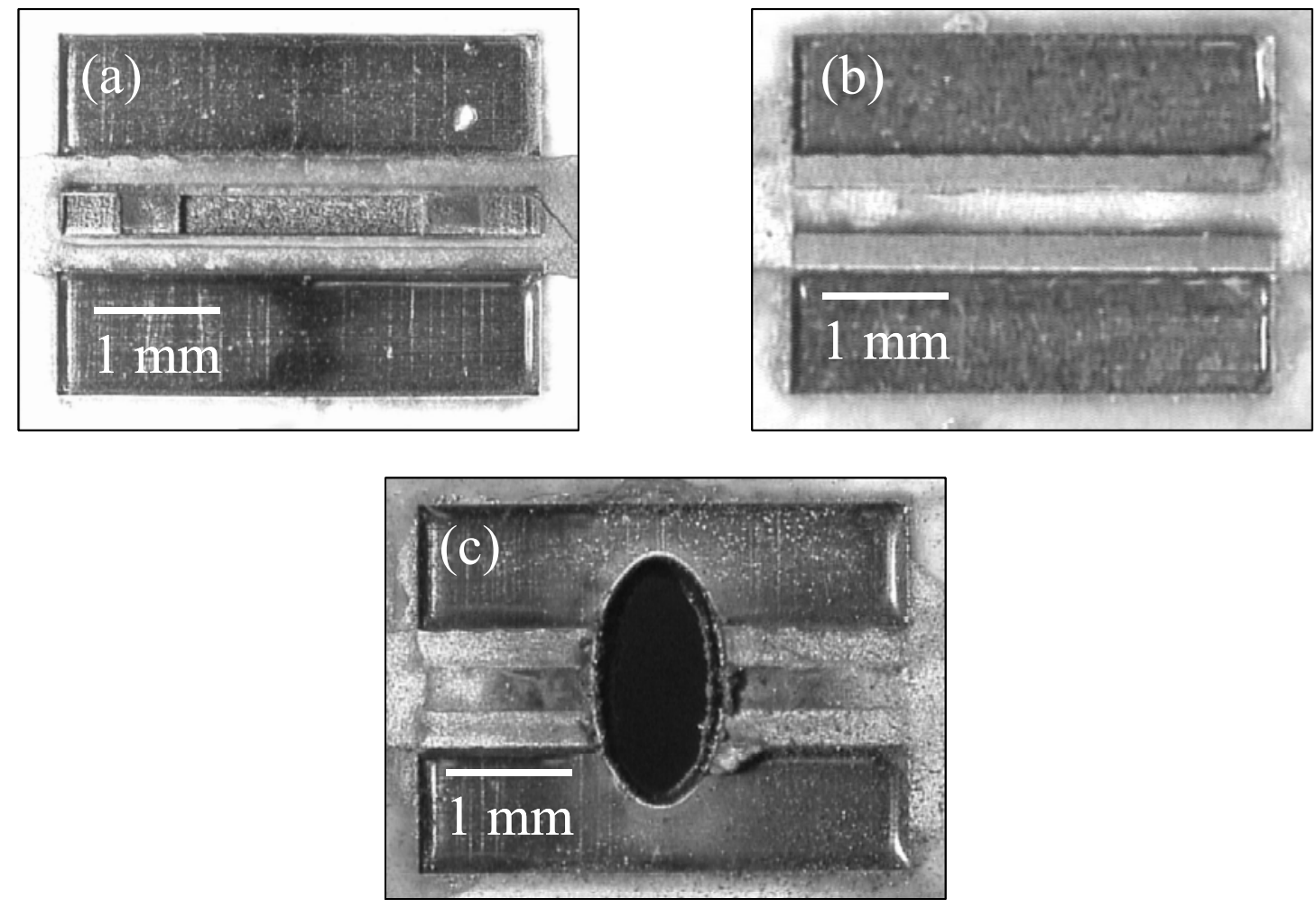

Figure 2: Optical micrograph of single defect sample. (a) laser micromachined terraces structure prior to silver deposition, (b) conformal silver deposition within groove, (c) final laser micromachined elliptical defect across silver conducting line.

Once the adhesive is set, a portion of the copper conductive lines is laser micromachined in the region of the initial defect. The copper is removed in an area of $4000 \mu \mathrm{m} \times 350 \mu \mathrm{m}$ without disturbing the underlying LCP or adhesive layer. Underlying layers are similarly removed over progressively smaller regions creating the stepped structure shown in figure 2a. Finally, the SPC layer is removed from the top of the copper lines by laser cleaning at lower fluences (15 $\left.\mathrm{J} / \mathrm{cm}^{2}\right)$.

A conductive silver-coated copper, epoxy based ink (Dupont CB230) is deposited by laser direct-write (LDW) over the stepped structure $[5,6]$ to fill in the laser micromachined region and make electrical contact to the Ti sample below (See figure $2 b$ ). LDW is able to conformally deposit material over such steps and has been shown to survive limited fatigue tests without failure or significant changes in electrical properties [7]. Due to the viscous nature of the conductive ink, small amounts of the ink are observed to overflow onto the top of the copper lines. This excess material is removed by laser trimming prior to the final baking of the sample which takes place at $70{ }^{\circ} \mathrm{C}$ for 1 hour in order to cure the conductive ink.

The final stage in sample preparation is to create the initial defects in the Ti sample. An elliptical through-hole defect (1 $\mathrm{mm} \times 2 \mathrm{~mm}$ ) is laser micromachined at the center of the conductive line as shown in figure 2c. The major axis of the ellipse is generated normal to the loading axis of the sample. The computer control positioning and translation of the sample enables the production of any desired initial defect structure. In these experiments, four defects were introduced 

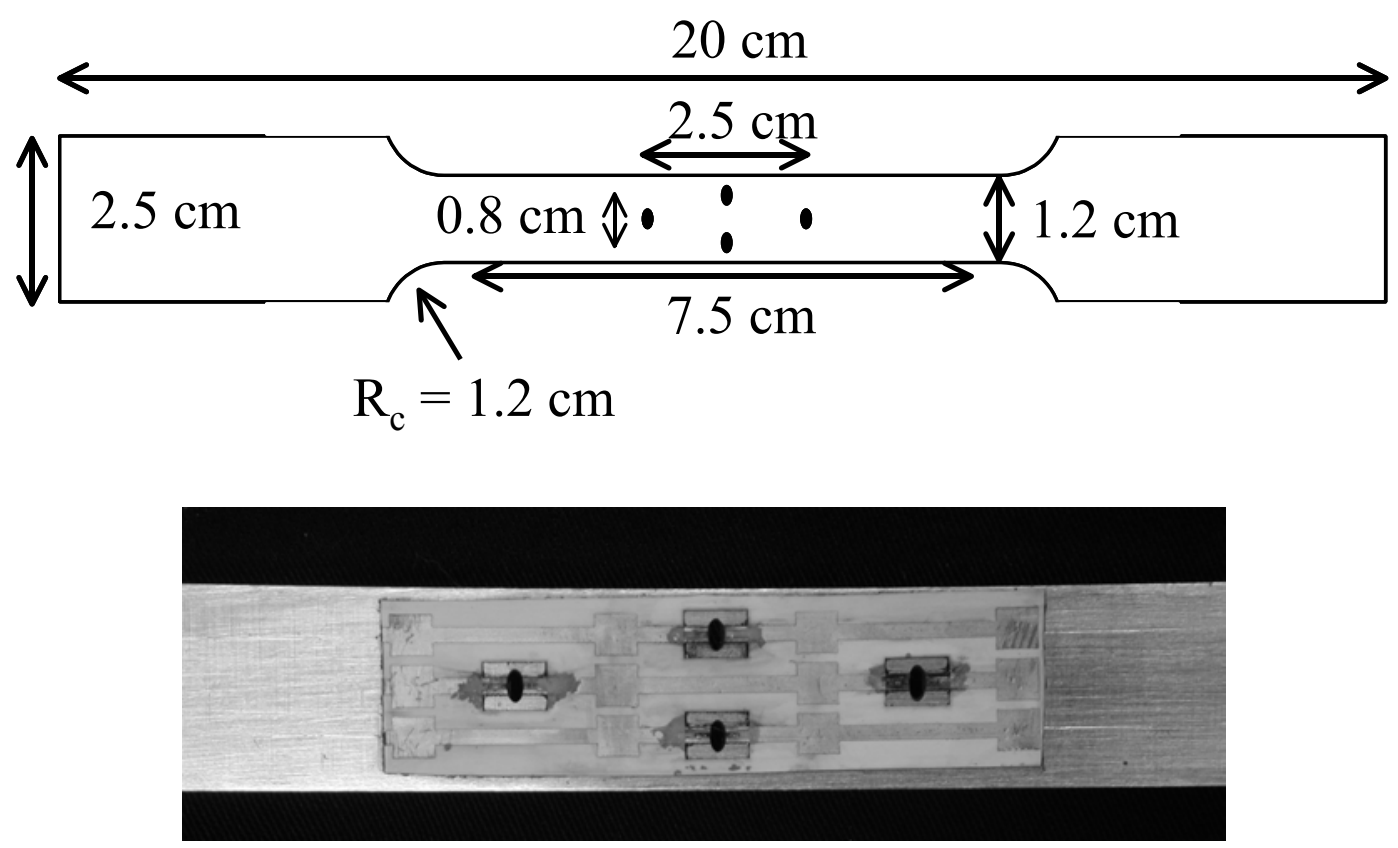

Figure 3: Dimensions and layout of the four laser micromachined defects on the dog bone sample.

to a single sample and arranged with dimensions as shown in figure 3. The final dimension of the contact pad at the base of the sample is $500 \mu \mathrm{m} \times 350 \mu \mathrm{m}$.

One of the main factors in controlling the sensitivity of the PD to changes in crack length is the ability to locate the leads as close as possible to the edge of the hole [2,4]. This laser based fabrication approach allows for precise, reproducible attachment of the potential drop leads at defects down to the mesoscale size. Such precision in lead attachment at such small defects is not currently possible using standard capacitive discharge welding techniques.

The sample is tested using an MTS servo-hydraulic loadframe. A constant 2 amp DC current is applied along the loading axis by leads attached on the specimen's loading section. The potential drop is measured using a Stanford Research System low-noise preamplifier (Model SR560) with a gain of 100 and a low pass filter setting at $10 \mathrm{~Hz}$. At the start of the test, the measured potential drop is approximately $1 \mathrm{mV}$ for three of holes in the array, and $10 \mathrm{mV}$ at the fourth hole. The potential drop voltage at all four holes is measured every 100 cycles up to a total of 1500 cycles. Subsequently, data is acquired every 250 cycles until specimen failure. The polarity of the current is reversed at each measurement point, producing two potential drop measurements at each condition. These two potential drop readings are averaged in order to eliminate errors due to changes in thermoelectric potential with temperature fluctuations.

Predictions of the fatigue test loading conditions needed to induce fatigue crack initiation in 10000 to 100000 cycles were made. Based on the stress concentration factors of the elliptical holes and an existing fatigue data for Ti-6Al-4V sheet, an initial maximum load, $\mathrm{P}_{\max }$, of $1330 \mathrm{~N}(300 \mathrm{lbs})$ was selected. The maximum load was increased after 10000 cycles to $1780 \mathrm{~N}$ ( $400 \mathrm{lbs}), 15000$ cycles to $2220 \mathrm{~N}$ (500 lbs), 175000 cycles to $2670 \mathrm{~N}$ (600 lbs) and 20000 cycles to $3110 \mathrm{~N}$ (700 lbs). A standard R-value $\left(\mathrm{P}_{\min } / \mathrm{P}_{\max }\right)$ of 0.1 was selected to keep the specimen in tension for the entire test. This R-value was kept constant for all loading conditions. 


\section{RESULTS}

Figure 4 shows a plot of the normalized voltage drop as a function of the cycle number for the sample described above. For this plot, the measured voltage drop was normalized by the initial voltage drop prior to loading. As expected, we see an initially flat response over the first 15-18 thousand cycles. At this cyclic level, an increase in the average voltage occurs, which corresponds to the nucleation of a crack at the edge of the ellipse major axis of this defect. After further cycling, the crack propagates, which is indicated by the rapidly increasing voltage drop. Ultimately, the crack begins to propagate in an unstable mode, leading to failure of the specimen. Similar profiles are obtained from the other defects on the dog bone sample.

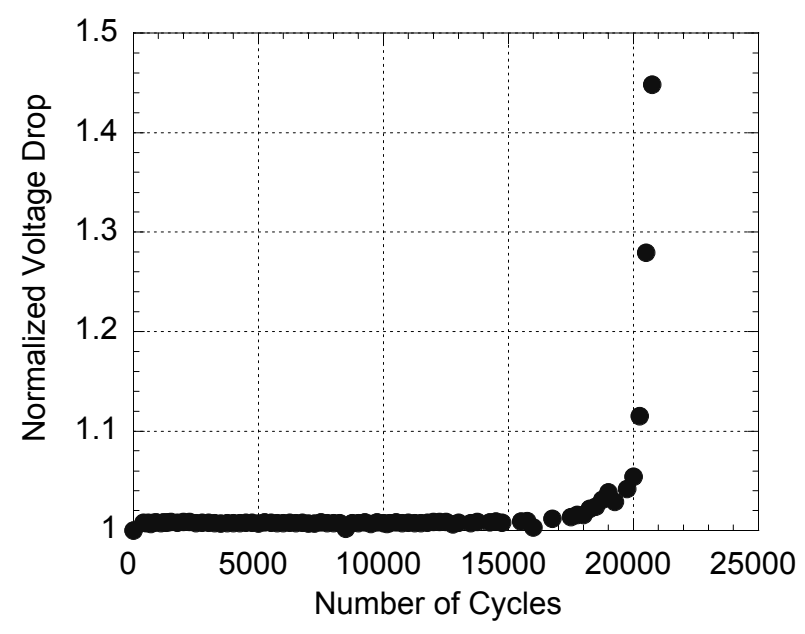

Figure 4: Normalized voltage drop as a function of cycle number

The optical image of the sample surface shown in figure 5 demonstrates typical features of fatigue failure. The cracks propagating from the edge of the initial defect give produce a straight line across the fracture surface. Based on this image the crack propagation occurred for $1 \mathrm{~mm}$. Following this region, the sample fractured as indicated by the jagged fracture surface. One of the most important features in this image is that the LDW silver line on the specimen remained intact over the course of the experiment. The lines show no signs of delamination or cracking that could lead to electrical changes to the potential drop study.

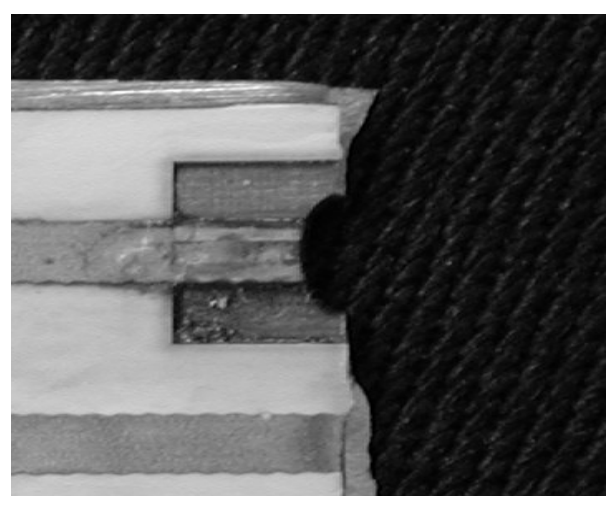

Figure 5: Top-view picture of the fractured specimen 


\section{SUMMARY}

We have shown the ability to generate addressable arrays of controlled defects for fatigue studies that can be monitored through local DC potential drop. Laser based techniques are used to deposit conductor lines on the titanium sample and create initial defects in intimate contact with the electrical leads. Preliminary tests demonstrate that the deposited conductors remain intact over the course of the experiment providing a robust measurement for the changing voltage drop across the defect. The observed increase in voltage drop indicates the nucleation and growth of cracks initiating along the major axis of the elliptical defect leading to ultimate fracture of the specimen. Future work will lead to a detailed calculation and measurement of real time crack lengths on multiple defects using this technique.

\section{REFERENCES}

1. Dowling, N., Mechanical Behavior of Materials, $2^{\text {nd }}$ Ed., New York, Prentice-Hall, 1999.

2. Suresh, S., Fatigue of Materials, $2^{\text {nd }}$ Ed., Cambridge; New York: Cambridge University Press, 1998.

3. Little, R.E., Manual on Statistical Planning and Analysis for Fatigue Experiments, ASTM STP 588, American Society for Testing and Materials, Philadelphia, PA, 1975.

4. ASTM Annual Book of Standards Vol. 03.01, Standard E-647, Measurement of Fatigue Crack Growth Rates, American Society for Testing and Materials, Philadelphia, PA, 2003.

5. Chrisey, D.B, McGill, A.M, and Pique, A. "Matrix assisted pulsed laser evaporation direct write", US Patent \#6177151, 2001

6. A. Piqué, D.B. Chrisey, R.C.Y. Auyeung, J. Fitz-Gerald, H.D. Wu, R.A. McGill, S. Lakeou, P.K. Wu, V. Nguyen, and M. Duignan, "A novel laser transfer process for direct-writing of electronic and sensor materials," Appl. Phys. A, 69, 279 (1999).

7. Pratap, B, Arnold, C.B, and Pique, A. "Depth and surface roughness control on laser micromachined polyimide for direct-write deposition" SPIE proceedings vol 4979, 217-226 (2003) 